\title{
Global assessment of innovative solutions to tackle marine litter
}

\author{
Nikoleta Bellou ${ }^{1 凶}$, Chiara Gambardellaㄹ, Konstantinos Karantzalos ${ }^{3}{ }^{3}$, João Gama Monteiro ${ }^{4}$, \\ João Canning-Clode ${ }^{4,5}$, Stephanie Kemna ${ }^{6}{ }^{6}$, Camilo A. Arrieta-Giron' ${ }^{1}$ and Carsten Lemmen (1) 1
}

\begin{abstract}
Marine litter is one of the most relevant pollution problems that our oceans are facing today. Marine litter in our oceans is a major threat to a sustainable planet. Here, we provide a comprehensive analysis of cutting-edge solutions developed globally to prevent, monitor and clean marine litter. Prevention in this research includes only innovative solutions to prevent litter entering oceans and seas rather than interventions such as waste reduction and recycling. On the basis of extensive search and data compilation, our analysis reveals that information is dispersed across platforms and is not easily accessible. In total, 177 solutions-the equivalent to $<0.9 \%$ of the search hits-fulfilled our validation criteria and were evaluated. Most solutions $(n=106$, $60 \%)$ primarily address monitoring and were developed during the past 3 years, with the scientific community being the key driver. Few solutions reached mature technical readiness and market availability, while none were validated for efficiency and environmental impact. Looking ahead, we elaborate on the limitations of the existing solutions, the challenges of developing new solutions, and provide recommendations for funding schemes and policy instruments to prevent, monitor and clean marine litter globally. In doing so, we encourage researchers, innovators and policy-makers worldwide to act towards achieving and sustaining a cleaner ocean for future generations.
\end{abstract}

T he quasi-exponential increase in marine litter or debris since the 1980s has been reported from as early as the early 1990 s $^{1}$. Two decades later, marine litter was already considered to be one of the most relevant pollution problems that our oceans and planet are facing ${ }^{2,3}$. Between 1990 and 2015, about 26-91 million tonnes of litter entered our ocean ${ }^{4}$, most of which was plastics $(61-87 \%)^{5,6}$. Although "by the end of the 1980 s, most impacts of marine litter were reasonably understood, and attention shifted to seeking effective solutions"7, it took more than 30 years to recognize marine litter as an urgent global issue for a healthy and sustainable planet, and to adopt it in policy instruments ${ }^{8,9}$. Meanwhile, a growing number of scientists and studies have highlighted its impacts on biota, socioeconomy and human health ${ }^{10-12}$.

With this increasing accumulation of marine litter, societies, policy-makers and industry now seem to understand the importance of developing and deploying solutions for tackling marine litter. These solutions should be effective and sustainable, including innovative technologies and new methods to prevent, monitor and clean (PMC) our seas and oceans. Despite the variety of literature on marine litter ${ }^{10-12}$, impacts, its occurrence, monitoring and treatment ${ }^{13-15}$, there is presently no integrative evaluation of proposed solutions addressing marine litter. To fill this gap, we present a comprehensive global assessment of innovative solutions, including methods, technologies and operational deployments, which have been or are presently being developed to prevent more litter from entering our oceans and seas, monitor litter and clean the already accumulated litter (Fig. 1). Global, national or regional initiatives and relevant activities that do not present, design, develop and deploy specific methods, innovative technologies and solutions related to the functions PMC were not assessed as these were out of the scope of this research.

An extensive review was performed based on citation databases, EU, US and Asian funding web portals and databases, crowdfunding platforms, as well as web searches and professional networks (Supplementary Fig. 1). The classification of innovative solutions was based on the PMC functions. Additional information was collected for each solution and taken into account. It is worth mentioning that a solution may tackle (and has therefore been classified) different litter sizes and application areas concurrently. All queries and analysis followed a rigorous approach by using consistent, harmonized criteria for retrieving/identifying innovative solutions by filtering all results against these criteria, and by further analysing and assessing only the qualified ones.

Our study presents a comprehensive analysis, highlighting the advances made to date towards tackling marine litter in situ, including all relevant PMC solutions. Gaps and challenges were identified in already developed and ongoing engineering efforts. This research further suggests where a future focus could be set for the management of marine litter that is already contaminating our seas and oceans, to achieve and sustain a healthy ocean and planet.

\section{Results}

From almost 20,000 search results for PMC overall, only $0.9 \%$ $(n=177)$ validated against the innovative solutions criteria (Fig. $2 \mathrm{a}$ and Supplementary Tables 1 and 2).

How to find innovative marine litter solutions. Most qualified solutions were found on the Web of Science $(n=67)$, constituting less than $2 \%$ of the search (Fig. 2a). The second highest search return

'Institute of Coastal System-Analysis and Modeling, Helmholtz Zentrum Hereon, Geesthacht, Germany. ${ }^{2}$ CNR IAS-National Research Council, Institute for the Study of Anthropic Impacts and Sustainability in the Marine Environment, Genoa, Italy. ${ }^{3}$ Remote Sensing Laboratory, National Technical University of Athens, Athens, Greece. ${ }^{4}$ MARE-Marine and Environmental Sciences Centre, Agência Regional para o Desenvolvimento da Investigação Tecnologia e Inovação (ARDITI), Funchal, Madeira, Portugal. ${ }^{5}$ Smithsonian Environmental Research Center, Edgewater, MD, USA. ${ }^{6}$ Maritime Robotics AS, Trondheim, Norway. 凶e-mail: nikoleta.bellou@hereon.de 


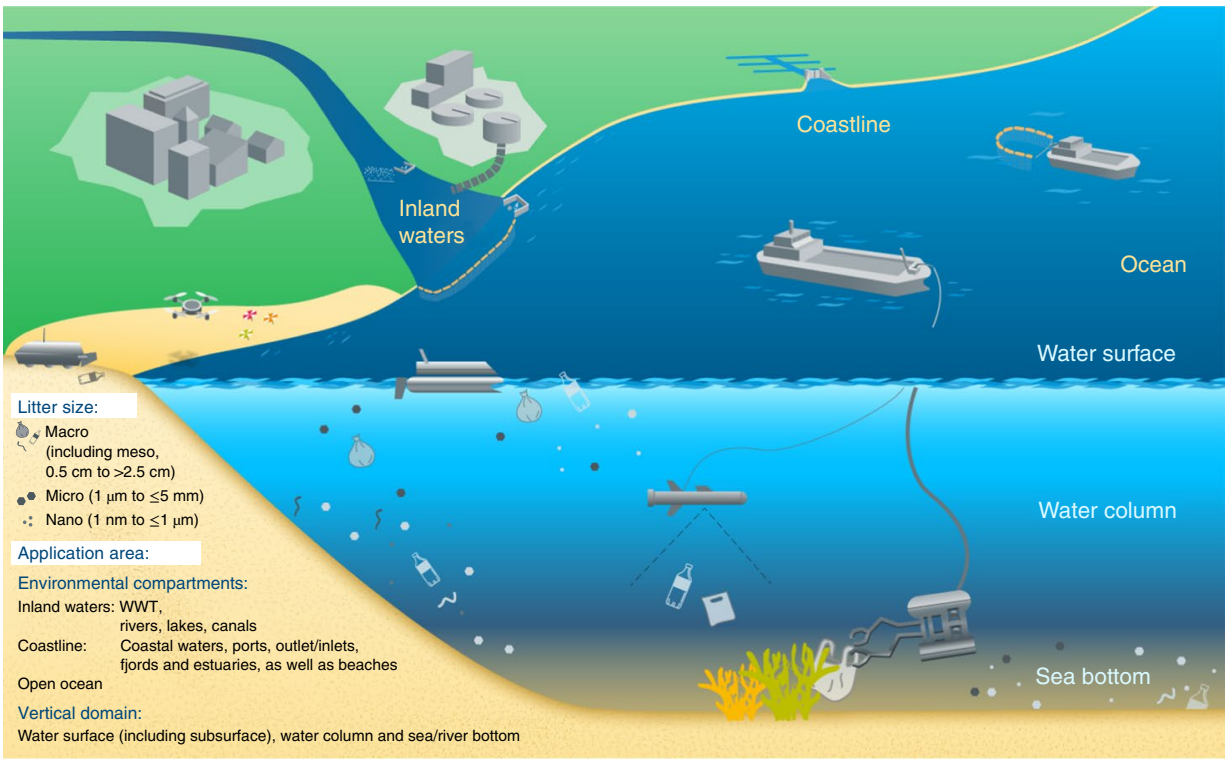

1. Prevention:

Technologies and/or methods that prevent marine litter from entering our oceans from rivers, including river mouths and discharge areas or from WWT plants. Preventive actions and measures (that is, best practices, citizen perceptions) to control marine litter issue were not included.

2. Monitoring:

Technologies and/or methods related to spatial and temporal assessment of marine litter occurrence, persistence, accumulation, distribution and/or contamination of coastal and/or marine environment. Moreover, those that monitor the presence and abundance, accumulation and distribution of marine litter were also included.

The function monitoring includes the sub-functions sampling, detection, modelling

Technologies and/or methods concerning the removal of marine litter, specifically in beaches, sea surface, water column, sea bottom in coastal and in open seas. 4. Multiuse

Technologies and/or methods that combine simultaneously at least two of the above PMC functions.

Fig. 1 | Overview of the classification of the innovative approaches according to their function and their areas of application. A summary of definitions for classifying each solution into a function, litter size and application area is included.

was through the authors' professional networks ( $n=45$, excluding those detected on other platforms). The EU Community Research and Development Information Service (CORDIS) repository registered the third highest number of solutions $(n=16)$. However, considering the ratio of qualified versus total hits, the European Space Agency (ESA) performed best $(32 \%, 9 / 28)$, followed by CORDIS $(25 \%, 16 / 63)$ and European Maritime and Fisheries Fund (EMFF) $(12 \%, 2 / 17)$, after their specific funding calls on 'marine litter innovation' (Supplementary Table 2). Easily accessible solutions through web search and crowdfunding platforms required the most screening. For example, only 12 out of 13,000 search hits on 5 crowdfunding platforms qualified-a $0.09 \%$ return.

Solutions by function and other categories. Most innovative solutions $(n=106,60 \%)$ addressed monitoring, divided into sampling $(n=19)$, detection $(n=50)$ and modelling $(n=37)$ (Supplementary Table 1). A recent analysis ${ }^{16}$ of EU projects on marine litter research development showed that monitoring-often together with policywas most represented, which is corroborated by our analysis.

Through monitoring, scientists have demonstrated that marine litter is present in all environmental compartments ${ }^{2,11,17}$. Marine litter is a growing concern particularly in coastal areas as the local economy is built around coastal marine resources and the blue economy ${ }^{18,19}$. With approximately $10 \%$ of the world's population in coastal areas relying on marine resources ${ }^{18}$, it is unsurprising that most PMC solutions target the coastline $(n=113$; Fig. $2 \mathrm{~b})$. Here, tourism has an important role, and marine litter can incur lost revenue $^{19}$. The latter could lead to $58 \%$ of the monitoring solutions focusing on the coast $(n=78), 25$ of which address litter on beaches, on which litter is often caused by tourism ${ }^{20}$.

Litter enters the marine environments mainly from land areas and rivers with estimates between 1.15 and 2.41 million tonnes of plastic entering the ocean every year from rivers alone ${ }^{21}$; less attention has been given to developing solutions for prevention $(n=33)$ in, for example, wastewater treatment (WWT). WWT plants in particular have been associated with micro- and nanoplastic pollution $^{22}$. Considering the toxic effects of microplastics on aquatic life $^{23}$, the low number of solutions for WWT $(n=6)$, which could act as control measures, does not indicate progress in reducing litter discharge. Neither does the low number in all other environmental compartments of inland waters (for example, rivers), which could aid prevention.

Although marine litter and its accumulation have been acknowledged as a major pollutant of our seas and oceans ${ }^{2,3,17}$, relatively few cleaning solutions have been developed $(n=30)$. Marine litter accumulates in all environmental compartments ${ }^{2,22,24}$, and the need for cleaning actions has been underlined and the value of clean-up efforts should be re-evaluated ${ }^{25}$. However, the most comprehensive global marine litter cleaning approach launched to date is manual beach clean-up actions, implemented mainly utilizing citizen campaigns ${ }^{14}$.

Multifunction solutions-with at least two PMC functionshave recently been suggested as a future approach on marine litter ${ }^{26}$. In our analysis, the first of eight multifunction solutions appeared in 2015 (Supplementary Table 1).

By analysing the application areas, independent of function, most solutions are shown to target litter on the water surface in marine and inland waters (Fig. 2b). The focus on water surfaces may be based on estimates that indicated that there is a minimum of 5.25 trillion litter particles floating in our oceans ${ }^{27}$. Overall, the solutions cover all environmental compartments; most of the solutions target coastlines $(n=113)$, followed by ocean $(n=73)$ and inland waters $(n=38)$. The high number of solutions for coastline surface waters and beach litter solutions may result from the impact that litter accumulation has on tourism revenue in coastal areas ${ }^{19}$. Our results show that, compared with the water surfaces, other vertical domains - such as the water column or sea bottom-are addressed less $(<34 \%)$. In contrast to the common perception that most litter floats at the surface, marine litter reaches the sea bottom. Even in the most remote marine environmental areas, for example, submarine canyons, the highest litter density can occur ${ }^{26}$. The sea bottom, compared with other domains, has only recently been included in research into marine litter solutions. Interestingly, multifunction solutions have been suggested recently for this domain ${ }^{26}$.

The categorization of marine litter according to size has been defined as a required reporting unit ${ }^{13}$. As there was an inconsistent use of litter size terms between different developers, the authors undertook an initial harmonization of litter size terms. The majority of solutions address macrolitter $(70 \% ; n=137)$ rather than microlitter $(27 \% ; n=54)$ or nanolitter $(3 \% ; n=6$; Fig. $2 \mathrm{c}$ and Supplementary Table 2). The extent of micro- and nanolitter contamination in the 
a

\author{
67
}

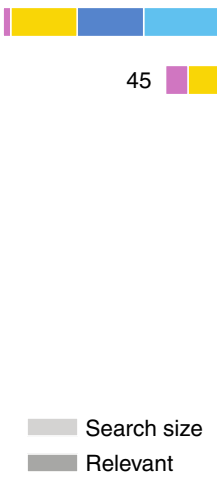



16

15

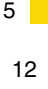

12

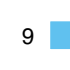

9

8

3
2
WoS

Network

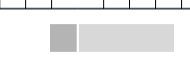

CORDIS

Web search

Crowd

ESA

NOAA

COBSEA

EMFF
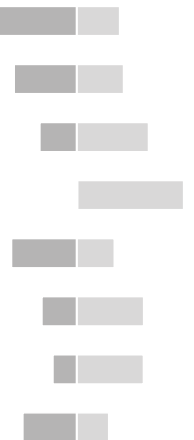

d

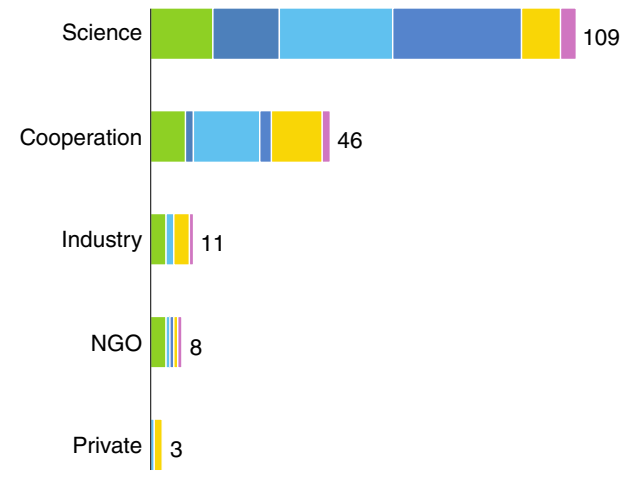

b

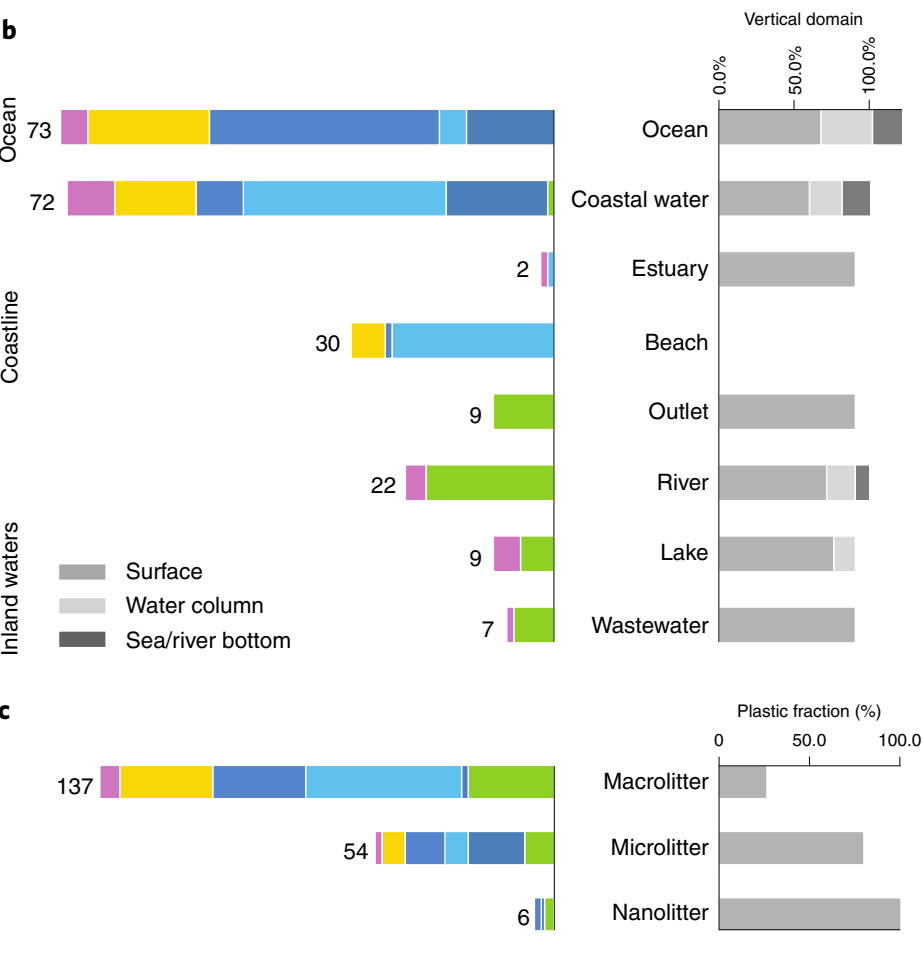

g

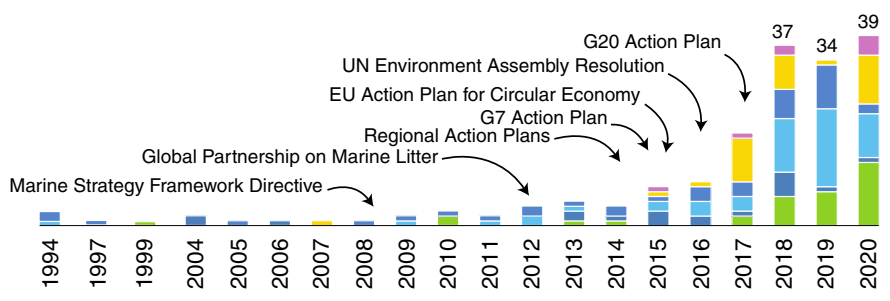

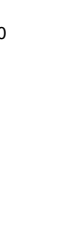

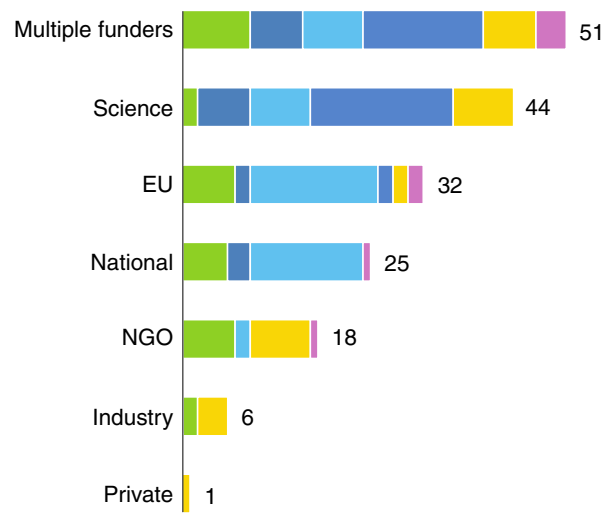

f

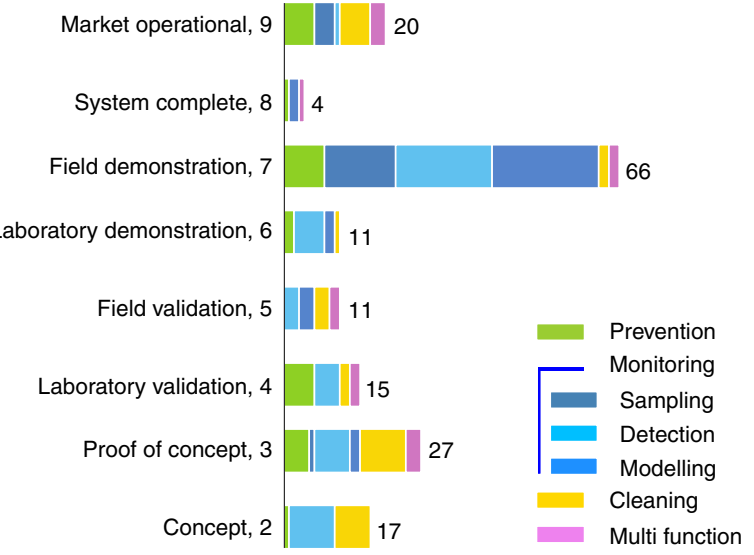

Fig. 2 | Analyses of search, domain, target, actors and actions by category. a, Search platform return for qualifying innovative solutions by category (colour), by ratio of innovative versus total (dark grey) and by total search corpus size (light grey). WoS, Web of Science. b, Ecosystems addressed from inland waters to the open ocean by category (colour) and by target depth (grey bars). c-g, Size and composition of marine litter by category (colour) and the fraction of plastic specifically addressed (grey) (c), initiator (d), funder (e), TRL (f) and timeline annotated with major policy actions and regulation (g). Cooperation and multiple denote any combination of at least two different initiators or funders. For $\mathbf{b}$ and $\mathbf{c}$, note that duplication is possible.

marine environment has only recently started to be documented ${ }^{28}$, resulting in few solutions for these sizes, primarily emphasizing plastic. By contrast, only $26 \%(n=36)$ of macrolitter solutions specifically target plastic, despite plastic being the most abundant material in the world's oceans ${ }^{29,30}$. The latter may be due to the lack of clear definitions and harmonized litter terminology between science, industry, policy-makers and technology developers. To enable metadata analyses and comparability of solutions, developers should document litter size and type on the basis of globally consistent and harmonized protocols, as used, for example, for marine litter 
Table 1 | The main technologies and methods used by the marine litter innovative solutions in accordance with their function

\begin{tabular}{|c|c|c|c|}
\hline Horizontal domain function & Surface & Water column & Bottom \\
\hline Prevention & Booms, traps, conveyor belt, filters & \multicolumn{2}{|c|}{ Autonomous underwater vehicles, ROVs, bottom: +Air } \\
\hline \multicolumn{4}{|l|}{ Monitoring } \\
\hline Detection & $\begin{array}{l}\text { Drones, aerial imagery, remote sensing, satellites, } \\
\text { multispectral, image analyses, unmanned aerial } \\
\text { vehicles }\end{array}$ & LIDAR & $\begin{array}{l}\text { Acoustic remote sensing, } \\
\text { sidescan sonar, infrared, ROVs, } \\
\text { drop camera, video analysis, } \\
\text { annotation }\end{array}$ \\
\hline Cleaning & $\begin{array}{l}\text { Vessels (various types), nets, booms, water drones, } \\
\text { aquarobots, beach robots, vacuum systems }\end{array}$ & Floaters, nets, booms & ROVs, vessels (various types) \\
\hline
\end{tabular}

ROVs, remote-operated vehicles; GIS, Geographic Information System; LIDAR, Light Detection and Ranging.

monitoring activities in European seas under the Marine Strategy Framework Directive (MSFD) ${ }^{13}$.

Driving forces for innovative marine litter solutions. Innovation is key to progress, and has a major role in the blue economy. To initiate and stimulate the development of new solutions or improve existing ones, and to integrate them into marine litter management, it is crucial to understand who is driving the change.

Our findings show that the scientific community is driving most innovations (Fig. 2d), having initiated more than half of the developments $(n=109)$, most of which $(n=79)$ were scientific publications on monitoring, particularly modelling and detection. Particularly in European waters and coastal areas, this could be a result of the well-established MSFD programme ${ }^{13}$, which is a key driver for marine litter monitoring studies in Europe. On CORDIS, which represents a wide variety of scientific disciplines as well as industry, the solutions found covered all functions. By contrast, only a few solutions-covering prevention, monitoring-detection and cleaning-were available on more-accessible platforms (web search and crowdfunding). Solutions found on the specialized platforms ESA and EMFF were limited to monitoring, especially detection, and similarly for the National Oceanic and Atmospheric Administration (NOAA) and Coordinating Body on the Seas of East Asia (COBSEA), although these platforms represent a large variety of disciplines.

Cooperations between scientists and other stakeholders (private, industry, non-governmental organizations (NGOs)) have initiated $26 \%$ of the solutions $(n=46)$. From 2015 onwards, industry, NGOs and private players have become more involved in the development of innovative solutions. However, the industry and private sectors initiated a few solutions by themselves. Cooperations are uniformly spread among the functions. Industry invested equally in prevention and monitoring, whereas NGOs initiated more prevention solutions.

More than half of the solutions $(n=101)$ were funded by scientific institutes or the EU and national funds (Fig. 2e). Almost a third was funded through cooperation between different funders, while private and industrial funds by themselves contributed together to only $4 \%$. Importantly, the scientific community has many funding sources (that is, regional, national, international and private). Although the scientific community's interest in marine litter research primarily started in the past decade ${ }^{7}$, it has strongly driven research and innovation on marine litter solutions, especially until 2014. Since then, there has been a shift and all initiators have shown an increased interest, leading to a quasi-exponential increase in the number of solutions as industry, NGOs and the private sector-initiated solutions.

Simultaneously, funding from all sources increased (Fig. 2e). The use of multiple funding sources for single solutions, as well as industry funds, showed a large increase since 2014. EU funding showed the highest increase over the past 6 years, thanks to different funding programs, such as Horizon2020. This increase in funding is also reflected in the total number of solutions and, therefore, in all functions. Between 1994 and 2014, only 17\% $(n=31)$ of the solutions were developed, while the remaining 146 were developed since then (Fig. $2 \mathrm{f}$ and Supplementary Table 2).

Shifts in policy and focus can also be ascertained by inspecting how the scope of the solutions changed over time (Fig. 2g, Supplementary Table 2 and Supplementary Fig. 2). Before 2014, almost $82 \%$ of the solutions focused on monitoring $(n=25)$, probably influenced by the MSFD, which provided funding for the assessment of the Good Environmental Status (GES) in European Seas. Since 2016, as regional Action Plans were created and the 'G20 Action Plan on Marine Litter' was adopted a year later, there has been a quasi-exponential increase in the number of solutions globally. In fact, it doubled from 2016 to 2017 and again from 2017 to 2018 . In the past 4 years alone, up to May 2020, 73\% of the recorded solutions were launched $(n=129)$. Furthermore, since the launch of the Regional Action Plans, an increasing proportion of solutions appears in other functions than monitoring, suggesting a shift from diagnosing to solving the problems.

The maturity of the marine litter solutions. An analysis of the reported and assessed technology readiness levels (TRL; Fig. 2f) revealed that most solutions were technically immature. In particular, 70 out of the 177 solutions have not been demonstrated in relevant operational environments $(\mathrm{TRL}<6)$, while 17 were still at a conceptual stage $(\mathrm{TRL}<3)$. Monitoring solutions have been documented the longest, but are still mostly at field demonstration levels (TRL 6-7) because the scientific community as their main initiator does not usually bring solutions to the market.

In 2004, the first solution reached TRL9, and it took almost ten more years for another solution to reach the same TRL (2013; Fig. 2f, Supplementary Table 1 and Supplementary Fig. 2). Only $11 \%$ of the solutions were operational (TRL9, $n=20: n=6$ cleaning, $n=5$ monitoring, $n=7$ prevention, $n=2$ multifunction; Fig. $2 \mathrm{f}$ ). Considering that innovation is important for sustainable businesses $^{31}$, this is quite a low percentage within 16 years. This number is especially low considering that efforts were already made to promote "Innovative business models for marine litter" ${ }^{32}$. For the TRL 9 


\section{Box 1 | Recommendations based on this analysis for future funding schemes and policy instruments to PMC marine litter globally}

1. Foster greater international cooperation, encourage cross-disciplinary collaboration and merge economic and scientific knowledge to evaluate existing and new solutions for prevention, monitoring and cleaning marine litter.

2. Create clear definitions and establish a harmonized terminology on litter sizes between science, industry, policy-makers and technology developers to make it easier to compare innovative solutions for addressing marine litter.

3. Create definitions and specific requirements to measure operational efficiency and environmental impact in environments in which solutions are deployed.

4. Re-evaluate the existing solutions according to recommendations 1,2 and 3 and select solutions that are good candidates for commercialization (bringing them to TRL9) and define the resulting gaps in terms of function and areas.

5. With surface waters being the key environmental compartments addressed by most prevention, monitoring and cleaning solutions, more emphasis should be given to solutions targeting the water column and sea bottom. Beaches and remote areas such as the open ocean and the deep sea should be taken into account as well.

6. Encourage new funding schemes for further developing existing and new solutions as well as for expanding the developments of solutions.

7. Strengthen litter management measures by integrating them into policy instruments that appoint responsibility for the prevention and cleaning of marine litter in national and international areas of accumulation.

8. Establish a global digital network of innovation for solutions on prevention, monitoring and cleaning litter that include key disciplines related to inventors, engineers, social scientists, economists and policy-makers.

9. Develop a digital integrated and engaging marine litter innovation database with efficiency and impact maps accessible for a broad range of stakeholders.

10. Develop a holistic sustainable approach for mitigating marine litter in accumulation areas or hotspots in various water environments by using innovative prevention, monitoring and cleaning solutions simultaneously.

solutions, cooperation between actors was the highest contributor $(n=8)$, followed by science $(n=5)$, NGOs $(n=4)$ and industry $(n=3)$. Most of the cleaning solutions arose from cooperations (Supplementary Table 1), while the scientific community developed most of the monitoring solutions and NGOs initiated most of the prevention solutions. This indicates that each function is addressed by different actors in marine litter management.

A discussion of the technical details of each solution was not in the scope of this Analysis, but it is worth mentioning that there is often an overlap or repetition of technology and methodological principles underlying different solutions, whereas new solutions are scarce (Table 1 and Supplementary Table 1). The duplication of efforts arises as different developers use similar approaches, and information on existing approaches can be difficult to find. For example, several similar types of booms or conveyor belts were suggested as parts of prevention solutions to collect marine litter before it enters our seas and oceans. Still, there is some indication that next generation approaches-using machine learning, robotics, big data analytics and modelling-are increasingly in focus for developers of marine litter solutions. Furthermore, there is a visible trend towards developing multifunction solutions, for example, simultaneous prevention and cleaning, together with recycling and reuse. Finally, we found that none of the solutions (at any TRL) provide information on their operational efficiency and environmental impact.

\section{Discussion}

Our analysis demonstrates that many PMC solutions need to be improved further to reach the market. The solutions developed to date seem to be promising, but some knowledge gaps and constraints remain, such as (1) a lack of harmonized size terminology use and data harmonization; (2) technological and methodological immaturity in terms of TRL; (3) a lack of more-diverse solutions; and (4) a lack of information on the efficiency and environmental impact of solutions.

Our data show that efforts have been made worldwide by various initiators and funders towards the development of innovative solutions for the PMC of marine litter. To date, the main focus has been on monitoring, mostly targeting water surfaces and beaches, while efforts are ongoing to identify and characterize the marine litter problem in all interlinked areas. Most of the monitoring solutions were initiated by the scientific community and do not surpass TRL 7. Future developments will probably consider technologies for advancing remote and autonomous monitoring ${ }^{33}$, implementing strategies to reduce $\operatorname{cost}^{13}$ and extending monitoring to all areas, including the deep-sea bottom. With monitoring dominating, the identification and characterization of the litter problem is currently the state of the art. Only $11 \%$ of the solutions $(n=20)$ have reached TRL 9, most remain at TRL 7. The need for bringing more solutions to the market could be met by creating more funding schemes that enable new startups or cooperation between the scientific community and industry to hand over prototype solutions to commercial product providers.

None of the current solutions provided information on their environmental impact or efficiency. These two aspects have only recently been taken up. One study highlighted that, for the evaluated beach cleaning methods, more empirical studies are required ${ }^{34}$. In another study, the efficiency of solutions regarding ocean clean-up devices and river barriers was evaluated using modelling tools ${ }^{35}$. Their results showed that it would take over 100 years to remove $5 \%$ of ocean plastics when using ocean clean-up devices stand-alone. Considering that inaction to address marine litter has both environmental and socioeconomic costs ${ }^{18,36}$, a combination of reduced plastic emissions and increased removal has been suggested as the only way to free the ocean of plastic waste ${ }^{35}$ and reach a healthy, sustainable state for our oceans and the planet.

Although several instruments are tackling marine litter pollution at the international, national and regional levels ${ }^{37}$, the responsibility for cleaning accumulated litter or for preventing more litter from entering our seas and oceans is still not defined. By contrast, monitoring is a required aspect of the MSFD, lying in the national responsibility of EU member states ${ }^{13,38}$. Although the EU has funded the development of almost $18 \%(n=32)$ of the solutions (Fig. 2e), including those for cleaning, it is stated under the EU 'Directive defines GES' for 'Descriptor 10: Marine Litter' that “Cleaning up the oceans is one option, it is however not the most efficient method against marine litter" and suggests to emphasize tackling the marine litter problem at its source ${ }^{39}$. Although the accumulation of marine litter is still continuing and its impact is undoubtable, recent EU and global strategies and policies have focused on reducing, banning or 
reusing/recycling single-use plastic ${ }^{37,40,41}$, rather than finding environmentally and financially sustainable solutions on further PMC. The 'Osaka Blue Ocean Vision' in 2019 opened the floor to PMC innovation under the G20 Marine Litter Action Plan framework, presenting the 'MARINE Initiative ${ }^{42}$. Actions on information and data sharing, as well as the integration of Innovative PMC Solutions into policies should be on the agenda for future global marine litter management events.

For solutions to get a market niche, the already defined business model barriers and the low customer buy-in ${ }^{43}$ need to be overcome. More importantly, the matter of solving the PCM responsibility on international, national and regional policy levels should be addressed. The gap in policy instruments regarding the responsibility for prevention and cleaning ${ }^{8,9,37}$ needs to be filled, as defined for monitoring marine litter, for example, in the EU through the MSFD ${ }^{38}$. As our seas and oceans do not have borders in several geographical regions, marine litter accumulation occurs more in regions without a clear PMC responsibility. The remoteness of litter accumulation in open oceans inhibits responsibility ${ }^{44}$. Furthermore, some coastal municipalities have much higher cleaning costs than average $\mathrm{e}^{45}$ and the willingness of citizens to contribute to the cleaning costs is area dependent ${ }^{36}$. However, cleaning marine litter should be integrated into current management policies, independently of its financial $\operatorname{cost}^{25}$, by considering the aspects of efficiency and environmental impact of the solutions ${ }^{34}$. Presently, the plastic production and the resulting waste accumulates faster than the steps taken towards innovative solutions to help reduce it and to improve the environmental status. Moreover, it was estimated that the amount of plastic reaching the ocean will peak in 2029 (ref. ${ }^{35}$ ), originating from land-based, riverine and ocean-based sources ${ }^{21,46,47}$. There is a global need for stronger actions and strategies from policy-makers and stakeholders to tackle marine litter ${ }^{25,48}$, which requires substantial progress in innovation, including marine litter solutions on PMC.

Finally, to achieve a significant and globally sustainable decline of marine litter, the present analysis of existing marine litter solutions provides ten recommendations (Box 1) for future funding schemes and policy instruments globally. We encourage the existing and new players in the development of PMC solutions to take this work further, to bring solutions to TRL 9 and solve the marine litter problem by also integrating PMC solutions into marine litter management. Only then can we achieve and sustain healthy oceans and a healthy planet for all.

\section{Methods}

An extensive search for innovative marine litter solutions was performed by screening various platforms between March and May 2020. The classification of innovative solutions was based on the function of each solution. A consistent approach was achieved by defining harmonized criteria for identifying innovative solutions found across all platforms. All search hits were evaluated on the basis of these predefined criteria. Additional information was collected only in cases in which the solution qualified according to all of the selection criteria. Systematic analyses were implemented on only qualifying solutions, where solutions could appear in the different categories in accordance with the additional information collected.

An overview of the main steps taken both for the selection and validation is provided in Supplementary Fig. 1.

Search platform. The publisher-independent global citation database Web of Science was consulted, together with research and development efforts funded by various sources, including the European Commission's funding web portals (CORDIS, ESA and EMFF), American and Asian databases (NOAA and COBSEA), and crowdfunding/makerspace platforms (Kickstarter, Indiegogo, Causes, Patreon, GoFundMe, CircleUp and ConservationX). Further search tools, such as professional networks and knowledge of the authors, Google search, global marine litter events and websites, were used to complement the results.

Definition of functions. To ensure consistency, the following definition statements for the functions of the operational solutions were used. Only solutions that target operational/in situ use were considered, while actions or initiatives that include citizen science campaigns were discarded (see the 'Selection criteria').

Prevention. Technologies and/or methods that prevent marine litter from entering our oceans from rivers, including river mouths and discharge areas, or from WWT plants. Preventive actions and measures, for example, best practices and citizen perceptions, were not included.

Monitoring. Technologies and/or methods related to the spatial and temporal assessment of marine litter occurrence, persistence, accumulation, distribution and/or contamination of coastal and/or marine environments. Moreover, those that monitor the presence and abundance, accumulation and distribution of marine litter were also included. The function monitoring includes the subfunctions sampling, detection and modelling.

Sampling. Innovative, automated and cost-effective technologies and/or methods, or advancements of traditional methods (for example, nets and pumps) in terms of efficiency and/or time reduction, and research outputs for field sample collection to monitor marine litter in beaches and sea water (including sea surface, water column and sea bottom). Outputs that were not included were as follows: (1) traditional methods to collect marine litter (for example, nets, box corer and grabber), as well as marine litter samples found/ingested in marine biota; and (2) technologies/methods related to sorting, identification or characterization of plastic polymers

Detection. Monitoring through supervised, semi-automated or automated

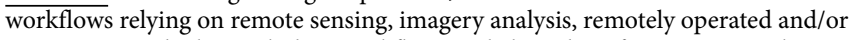
autonomous vehicles, including workflows and algorithms for imagery analysis, innovative methodologies and new technologies including models and AI methods implemented to detect and/or map marine litter. Query results on visual monitoring from ships or manual beach surveys were not included.

Modelling. Monitoring through modelling, including statistical models, increasingly supported by AI methods, particle tracking, predominantly $2 \mathrm{D}$ and 3D approaches, buoyancy processes that include plastic density variety, (dis) aggregation and biofouling processes, and budget models. Modelling solutions restricted to inland waters were not included.

Cleaning. Technologies and/or methods concerning the removal of marine litter, specifically in beaches, sea surface, water column, sea bottom in coastal and in open seas.

Multifunction. Technologies and/or methods that simultaneously combine at least two of the above functions.

Selection criteria. Independent searches were implemented by one author with expertise in each function (prevention, monitoring or cleaning) to achieve a harmonized and consistent approach in identifying innovative solutions (technologies/methods) on the different platforms. Each author applied common criteria, which are listed below, to the search returns. Each result was then analysed for the extent to which it contributes, and for the relevance to the function. The results were evaluated on the basis of the following screening procedure criteria. Only solutions that fulfil all of the criteria were included in the present analysis:

- Criteria 1. Does the solution have a description (title and abstract) that is clearly relevant to in situ PMC functions, moreover in relation to its respective definition statement? (Yes/No)

- Criteria 2. Does the solution target at least one type of marine litter or a specific litter size in the corresponding application area? (Yes/No)

- Criteria 3. Is the solution new/innovative in terms of concept, methodology, technology or other engineering aspects? (Yes/No)

- Criteria 4. Does the solution achieve increased efficiency and/or performance compared with manual/previous solutions? (Yes/No)

- Criteria 5. Has the solution been designed towards achieving a higher TRL and/or operational use/deployment? (Yes/No)

Additional information. Additional information that was collected for each solution included the following: application area, littersize, litter target, TRL, initiator, funder, technology and year. An overview of all data collected for the analyses is provided as in the Supplementary Information in accordance with the function of the solution.

Application area. The application area is the area in which each solution can be applied and includes the following two aspects: the environmental compartment and the vertical domain.

Environmental compartments. The environmental compartments were categorized as inland waters (including, WWT, rivers, lakes and canals), coastline (including coastal waters, ports, outlet/inlets, fjords and estuaries, and beaches) and open ocean. 
Vertical domains. Vertical domains were distinguished as the water surface (including subsurface), water column and sea/river bottom.

Litter size. The litter size was categorized according to the Directive Guidance on Monitoring of Marine Litter ${ }^{13,49}$ as macro (including meso, $>5 \mathrm{~mm}$ ), micro ( $1 \mu \mathrm{m}$ to $\leq 5 \mathrm{~mm}$ ) and nano ( $1 \mathrm{~nm}$ to $\leq 1 \mu \mathrm{m}$ ) and combined with the information to target specifically plastic material or general litter.

TRL. The TRL was ascribed according to H2020 Work Program 2014-2015 (Commission Decision C(2014)4995) ( $^{50}$ and the (earliest) publication year recorded In particular, for monitoring and cleaning solutions, a TRL of above 5 indicates that, for example, the deployed technologies (including hardware, software and/ or integrated systems) were tested and validated in specific, geographically constrained areas or even in simulations, while a TRL of above 6 indicates that the deployed solutions were validated in operational environments testing in full-scale efficiency and scalability.

Initiator. The initiator was found by affiliation of project or collaboration partners.

Funder. The funder was identified from funding statements or the acknowledgements section. From the short description of the solution, we extracted keywords that describe the main technologies used in each solution.

Year. The year reflects either the year of publication or the year of the TRL.

Main technology. For each evaluated solution, the main technological aspects were studied and analysed, including the main sensing and engineering components, the overall architecture including hardware, software and communications (when required) as well as the different components and possible dependencies with other systems of solutions.

Semantic analysis of selected results. For each solution that fulfilled the criteria we analysed the collected data according to the additional information collected, including the environmental application location, the target material and size, the TRL and year, the initiator and funder of the solution, and keywords describing the technical solution. Considering one solution may address more than one litter size (for example, macro, micro) and application area (for example, water surface, water column), duplicates can be observed in the results of this Analysis, increasing the total solution number. An automated data workflow for the analysis and graphical representation of the data was conducted ${ }^{51}$ using open source software, including Python, Seaborn, LaTeX and Inkscape ${ }^{52-55}$.

Implementation of search according to each platform. Web of Science. We queried 'All databases' covering the period 1900-2020 in the English language. As our first-level selection criterion, we used 'marine' and 'litter or debris'. Second-level queries were formulated by adding the terms 'prevention', 'monitoring' or 'cleaning'. To account for heterogeneous functional morphemes, we used a regular expression syntax on Web of Science and replaced the affix with an asterisk, for example, 'clean' to denote any word starting with the letters c, l, e, a, n followed by zero or more arbitrary letters, therefore including the words 'clean' and 'cleaning'.

For the 'prevention' results, the search was refined by adding the third-level terms 'robotics', 'innovation', 'automated' or 'solution' (with search expressions 'robot ${ }^{*}$ ', 'innovat ${ }^{*}$ ', 'automat ${ }^{* \text { ' }}$ and 'solution ${ }^{*}$ ', respectively). For the 'monitoring' results, we refined the search by dividing them into 'detection', 'sampling' and 'modelling or simulation'. Among 'detection', the search was refined by using queries including a second-level criterion with any of the terms 'monitoring', 'detection' or 'distribution', all using affix regular expressions. As marine litter models and simulation tools are used to locate sources and destinations of litter, these were semantically categorized under monitoring-detection for the purpose of this review. However, typically, the keywords 'monitoring' and 'detection' are not used together in the literature on modelling marine litter-the search was performed by placing 'modelling or simulation' on a third level and not using 'detection'.

CORDIS. As a primary information source of EU-funded projects by the framework programmes for research and innovation (FP1 to Horizon 2020) from the European Commission, we used the CORDIS (https://cordis.europa.eu/) to identify funding towards marine litter issues. An initial search in CORDIS (https://cordis.europa.eu/search) used the term 'marine litter or debris' with a filter to include only 'projects'. The keywords mentioned for the Web of Science at first level (for example, prevention, monitoring) were chosen and selected.

ESA. The ESA Open Space Innovation Platform (https://ideas.esa.int/servlet/ hype/IMT?userAction=BrowseCurrentUser\&templateName $=$ MenuItem) was used to identify innovative solutions on the basis of remote sensing and Earth observation to be included within the monitoring-detection category. In 2019, the ESA launched a campaign in search of ideas towards the development of remote sensing of plastic marine litter. Ideas submitted and selected for further processing and supported by ESA were identified by exploring their Open Space Innovation Platform and searching for the term 'marine litter'.

EMFF. The EMFF was used to identify projects in the context of marine litter. The search tool within the EMFF site (https://ec.europa.eu/easme/en/emff-projects) was used without the use of the option 'Call identifier'. As the initial assessment of the key term 'marine litter' resulted in a very low number of hits, separate searches were performed using keywords 'marine', 'litter', 'plastic', 'prevention', 'monitoring' and 'cleaning'.

NOAA. The NOAA is a US governmental scientific agency that financially supports projects to understand and predict changes in climate, weather, oceans and coasts, and that seeks to conserve and manage coastal and marine ecosystems and resources. Its database (https://grantsonline.rdc.noaa.gov/) was used as the search engine for NOAA's grants. The terms 'marine litter' and 'marine debris' were applied to filter for relevant results using filter options 'Project Description' and 'Project Title'. For the keywords 'marine litter', there were no results, so all of the findings were associated with the term 'marine debris'. For all of the results, the 'associated amendments' that serve as subprojects of the general funding were considered and included if they fulfilled the criteria for selection.

COBSEA. The Asian database generated by the COBSEA and the National University of Singapore have gathered papers and projects that seek solutions on marine plastic pollution from over 13 Asian countries. This database is compiled in an online Excel file named 'Marine Litter Research Inventory-ASEAN+3' (www.gpmarinelitter.org/news/news/ cobsea-and-nus-launch-database-marine-litter-research-13-asian-countries) through which one has access to a Google Docs spreadsheet (https://docs.google. com/spreadsheets/d/1r4aCVQeCS1cj_Rhip82yVTNNnxkWDgFwbEIHCR_oASk/ edit\#gid=0, accessed May 2020), which includes information columns on, for example, methodologies, types of plastic, marine environments, year and authors. It was necessary to evaluate all of the referenced papers manually, as the metadata in the database were insufficient to assess relevance. The main columns used for selecting papers were 'Aim of Research', 'Methodologies' and 'Link to the source'.

Crowdfunding platforms. Crowdfunding refers to the idea of raising funds for a project or cause with the support of a large group of people, which has become particularly interesting with the advent of online crowdfunding platforms that increase access to a worldwide audience. This is a way for individuals, small businesses or scientists to obtain early-stage financial support for their ideas, as well as early marketing and allowing for media uptake. Paid crowdfunding platforms were excluded and only well-known platforms were included. The platforms selected were as follows: Kickstarter, Indiegogo, Patreon, GoFundMe, Causes, CircleUp and Conservation X. These were selected because they provided project rankings by funding and backers under the top three in their area. For creative projects, Kickstarter (https://www.kickstarter.com) and Indiegogo (https://www.indiegogo.com) were chosen; for personal crowdfunding and fundraising for nonprofit organizations, Patreon (https://www.patreon. com), GoFundMe (https://www.gofundme.com) and Causes (https://www. causes.com) were chosen; for equity, Crowdfunding CircleUp (https://circleup. com) was chosen; and, for a digital market platform, Conservation X (https:// conservationxlabs.com) was chosen. Differences between the search tool options within each crowdfunding platform and the limitations in using combined keyword search made it impossible to execute the exact same search on each platform. After an initial assessment of the crowdfunding platforms' search constraints, a different approach was used to ensure coherent and comparable results across platforms. Using the Google Search engine, two different online queries were conducted:

1. site:\{name of crowdfunding platform $\}$ marine (litter OR plastic OR plastics)

2. site:\{name of crowdfunding platform $\}$ marine (litter OR plastic OR plastics) (device)

Whenever a title or the short description of a project provided insights that innovative devices and or methods were presented for funding, the detailed description was read and information was extracted. If information was limited, additional information was gathered through an additional Google search using either the acronym of the device/method or information from the fundraiser as keywords. During screening, only projects that had achieved funding goals or had ongoing funding campaigns (at the time of the search implementation) were selected to be presented.

Web search. Further information relating to technologies and methods was compiled on the basis of conferences that the authors attended, as well as through their professional networks. Moreover, with the use of web search (Google) by using a combination of the keywords 'marine litter' (cleaning OR innovation), 'marine litter' (prevention OR innovation), 'marine litter' (monitoring OR sampling OR innovation) and 'marine litter' (monitoring OR detection OR innovation), the broader internet was screened for prevention, monitoring or cleaning technologies that might not have been found through the platform searches. Only the results 
that were defined by Google as the most relevant results were evaluated. Omitted entries were very similar to the already listed relevant ones.

Network. Once the systematic search in all of the previously mentioned platforms was implemented, the authors cross-checked whether solutions that they knew through their marine litter networks were listed as well. The authors' networks include solutions mentioned either in the scientific literature, developed in the lifetime of cooperative projects or by industry, NGO and private.

\section{Data availability}

The data supporting the findings of this study are available in the Supplementary Information. Additional information collected for the PMC solutions has been compiled into a separate dataset and uploaded to ZENODO ${ }^{56}$ with a temporary embargo. Any request for full access will be evaluated by the corresponding author.

Received: 14 December 2020; Accepted: 29 April 2021; Published online: 10 June 2021

\section{References}

1. Ryan, P. G. \& Moloney, C. L. Marine litter keeps increasing. Nature 361, 23 (1993).

2. Pham, C. K. et al. Marine litter distribution and density in European seas, from the shelves to deep basins. PLoS ONE 9, (2014); https://doi.org/10.1371/ journal.pone.0095839

3. Law, K. L. Plastics in the marine environment. Ann. Rev. Mar. Sci. 9, 205-229 (2017).

4. Laufkötter, C. et al. M. Marine plastic waste input between 1990-2015 and potential beaching scenarios. In Proc. EGU General Assembly 2020, EGU2020-18476 (2020); https://doi.org/10.5194/egusphere-egu2020-18476

5. Barboza, L. G. A. et al. in World Seas: An Environmental Evaluation Volume III: Ecological Issues and Environmental Impacts (ed. Sheppard, C.) 305-328 (Elsevier, 2019); https://doi.org/10.1016/B978-0-12-805052-1.00019-X

6. Bergmann, M., Tekman, M. B. \& Gutow, L. in Fate and Impact of Microplastics in Marine Ecosystems (eds Baztan, J. et al.) 106-107 (Elsevier, 2017); https://doi.org/10.1016/B978-0-12-812271-6.00104-6

7. Ryan, P. G. in Marine Anthropogenic Litter (eds Bergmann, M. et al.) 1-25 (Springer, 2015); https://doi.org/10.1007/978-3-319-16510-3_1

8. Marine Plastic Debris and Microplastics_Global Lessons and Research to Inspire Action and Guide Policy Change (United Nations Environment Programme, 2016); https://wedocs.unep.org/handle/20.500.11822/7720

9. Arroyo Schnell, A., Klein, N., Gómez, G. \& Sousa, J. National Marine Plastic Litter Policies in EU Member States: An Overview (IUCN, 2017); https://www. europarc.org/wp-content/uploads/2017/11/IUCN_National-marine-plastic litter-policies-in-EU-Member-States.pdf

10. Werner, S. et al. Harm Caused by Marine Litter. MSFD GES TG Marine Litter-Thematic Report (Publications Office of the European Union, 2016); https://doi.org/10.2788/690366

11. Agamuthu, P., Mehran, S., Norkhairah, A. \& Norkhairiyah, A. Marine debris: a review of impacts and global initiatives. Waste Manag. Res. 37, 987-1002 (2019)

12. Galgani, L., Beiras, R., Galgani, F., Panti, C. \& Borja, A. Editorial: impacts of marine litter. Front. Mar. Sci. 6, (2019); https://doi.org/10.3389/fmars. 2019.00208

13. Hanke, G. et al. MSFD Guidance on Monitoring of Marine Litter in European Seas EUR 26113 (Publications Office of the European Union, 2013); https://doi.org/10.2788/99475

14. Serra-Gonçalves, C., Lavers, J. L. \& Bond, A. L. Global review of beach debris monitoring and future recommendations. Environ. Sci. Technol. 53, 12158-12167 (2019).

15. Iñiguez, M. E., Conesa, J. A. \& Fullana, A. Marine debris occurrence and treatment: a review. Renew. Sustain. Energy Rev. 64, 394-402 (2016).

16. Maes, T., Perry, J., Alliji, K., Clarke, C. \& Birchenough, S. N. R. Shades of grey: marine litter research developments in Europe. Mar. Pollut. Bull. 146, 274-281 (2019).

17. Galgani, F., Hanke, G. \& Maes, T. in Marine Anthropogenic Litter (eds Bergmann, M. et al.) 29-56 (Springer, 2015); https://doi.org/10.1007/ 978-3-319-16510-3 2

18. Marine Litter Socio Economic Study (United Nations Environment Programme, 2017); https://wedocs.unep.org/bitstream/handle/20.500.11822/ 26014/Marinelitter_socioeco_study.pdf?sequence

19. Watkins, E. et al. Marine Litter: Socio-Economic Study. Scoping Report (IEEP, 2015). https://www.bundesregierung.de/resource/blob/975226/436888/a8d13d dc42b36d8d71048b3ee39dcfc0/2015-06-01-marine-litter-data.pdf? download $=1$

20. Wilson, S. P. \& Verlis, K. M. The ugly face of tourism: marine debris pollution linked to visitation in the southern Great Barrier Reef, Australia. Mar. Pollut. Bull. 117, 239-246 (2017).
21. Lebreton, L. C. M. et al. River plastic emissions to the world's oceans. Nat. Commun. 8, 15611 (2017).

22. Blair, R. M., Waldron, S., Phoenix, V. \& Gauchotte-Lindsay, C. Micro- and nanoplastic pollution of freshwater and wastewater treatment systems. Springer Sci. Rev. 5, 19-30 (2017).

23. Kögel, T., Bjorøy, Ø., Toto, B., Bienfait, A. M. \& Sanden, M. Micro- and nanoplastic toxicity on aquatic life: determining factors. Sci. Total Environ. 709, (2020); https://doi.org/10.1016/j.scitotenv.2019.136050

24. Cózar, A. et al. Plastic debris in the open ocean. Proc. Natl Acad. Sci. USA 111, 10239-10244 (2014).

25. Burt, A. J. et al. The costs of removing the unsanctioned import of marine plastic litter to small island states. Sci. Rep. 10, 14458 (2020).

26. Madricardo, F. et al. How to deal with seafloor marine litter: an overview of the state-of-the-art and future perspectives. Front. Mar. Sci. 7, https://doi. org/10.3389/fmars.2020.505134 (2020).

27. Eriksen, M. et al. Plastic pollution in the world's oceans: more than 5 trillion plastic pieces weighing over 250,000 tons afloat at sea. PLOS ONE 9, e111913 (2014).

28. Alimi, O. S., Farner Budarz, J., Hernandez, L. M. \& Tufenkji, N. Microplastics and nanoplastics in aquatic environments: aggregation, deposition, and enhanced contaminant transport. Environ. Sci. Technol. 52, 1704-1724 (2018)

29. Derraik, J. G. B. The pollution of the marine environment by plastic debris: a review. Mar. Pollut. Bull 117, 239-246 (2002)

30. Sheavly, S. B. \& Register, K. M. Marine debris \& plastics: environmental concerns, sources, impacts and solutions. J. Polym. Environ. 15, 301-305 (2007).

31. Teece, D. J. Business models, business strategy and innovation. Long Range Plann. 43, 172-194 (2010).

32. European Maritime Day: Ports and Coasts, Gateways to Maritime Growth. EMD Programme (European Commission, 2015); https://ec.europa.eu/ maritimeaffairs/content/european-maritime-day-2015-piraeus-exploringpotential-ports-and-coasts-maritime-growth_en

33. Conchubhair, D. O. et al. Joint effort among research infrastructures to quantify the impact of plastic debris in the ocean. Environ. Res. Lett. 14, 065001 (2019).

34. Zielinski, S., Botero, C. M. \& Yanes, A. To clean or not to clean? A critical review of beach cleaning methods and impacts. Mar. Pollut. Bull. 136, 390-401 (2019).

35. Hohn, S. et al. The long-term legacy of plastic mass production. Sci. Total Environ. 746, 141115 (2020)

36. Brouwer, R., Hadzhiyska, D., Ioakeimidis, C. \& Ouderdorp, H. The social costs of marine litter along European coasts. Ocean Coast. Manag. 138, 38-49 (2017).

37. Chen, C.-L. in Marine Anthropogenic Litter (eds Bergmann, M. et al.) 395-428 (Springer, 2015); https://doi.org/10.1007/978-3-319-16510-3 15

38. MSFD-Marine Strategy Framework Directive (European Commission, 2008, accessed Dec 2020); https://ec.europa.eu/environment/marine/eu-coast-andmarine-policy/marine-strategy-framework-directive/index en.htm

39. Good Environmental Status, Descriptor 10: Marine Litter (European Commission, 2020, accessed May 2021); https://ec.europa.eu/environment/ marine/good-environmental-status/descriptor-10/index_en.htm

40. Reducing Marine Litter: Action on Single Use Plastics and Fishing Gear SWD(2018), 76 (European Commission, 2018); https://ec.europa.eu/info/law/ better-regulation/have-your-say/initiatives/1502-Reducing-marine-litter-actio n-on-single-use-plastics-and-fishing-gear

41. Improving Resource Efficency to Combat Marine Plastic Litter (OECD, 2019); https://www.oecd.org/g20/summits/osaka/OECD-G20-Paper-ResourceEfficiency-and-Marine-Plastics.pdf

42. Japan's 'MARINE Initiative' Toward Realization of the Osaka Blue Ocean Vision (Ministry of Foreign Affairs of Japan, 2019, accessed Dec 2020); https://www. mofa.go.jp/ic/ge/page25e_000317.html

43. Dijkstra, H., van Beukering, P. \& Brouwer, R. Business models and sustainable plastic management: a systematic review of the literature. J. Clean. Prod. 258, 120967 (2020).

44. Van Sebille, E., England, M. H. \& Froyland, G. Origin, dynamics and evolution of ocean garbage patches from observed surface drifters. Environ. Res. Lett. 7, 044040 (2012).

45. Cruz, C. J. et al. Beach cleaning costs. Ocean Coast. Manag. 118, 105118 (2020).

46. Jambeck, J. R. et al. Plastic waste inputs from land into the ocean. Science 437, 768-771 (2015).

47. Browne, M. A. in Marine Anthropogenic Litter (eds Bergmann, M. et al.) 229-244 (Springer, 2015); https://doi.org/10.1007/978-3-319-16510-3_9

48. Canning-Clode, J., Sepúlveda, P., Almeida, S. \& Monteiro, J. Will COVID-19 containment and treatment measures drive shifts in marine litter pollution? Front. Mar. Sci. 7, 2018-2021 (2020).

49. Gago, J., Carretero, O., Filgueiras, A. V. \& Viñas, L. Synthetic microfibers in the marine environment: a review on their occurrence in seawater and sediments. Mar. Pollut. Bull. 127, 365-376 (2018). 
50. EC Horizon 2020 - Work Programme 2016-2017. General Annexes G. Technology readiness levels (TRL) (European Commission); https://ec.europa. eu/research/participants/data/ref/h2020/other/wp/2016_2017/annexes/ h2020-wp1617-annex-g-trl_en.pdf

51. Bellou N. et al. Data Analysis for Figure Creation: Global Assessment of Innovative Solutions to Tackle Marine Litter v.1.0.1 (Zenodo, 2021); https://doi. org/10.5281/zenodo.4674285

52. van Rossum, G. \& Drake, F. L. Python Reference Manual (CreateSpace, 2009).

53. Waskom, M. et al. mwaskom/seaborn v.0.11.0 (2020)

54. Lamport, L. LaTeX A Document Preparation System 2nd edn (Addison-Wesley, 1994).

55. Inkscape Project; https://inkscape.org

56. Bellou, N. et al. Dataset: Global Assessment of Innovative Solutions to tackle Marine Litter (Zenodo, 2021); https://doi.org/10.5281/zenodo.4548684

\section{Acknowledgements}

We thank all of the members of the BluePHOENIX consortium for their contribution to the overall BluePHOENIX concept. This work was supported in part by the HGF-EU Grant support (grant number 2020-006) for the BluePHOENIX proposal and contributes to the PoF IV Program 'Changing Earth-Sustaining our Future, Topic 4 'Coastal Transition Zones under Natural and Human Pressure' of the Helmholtz Association.

\section{Author contributions}

N.B. conceived and designed the analysis; planned and supervised the work; collected, together with the co-authors, the data for all functions in all platforms and revised them, as well as revised them according to the criteria; outlined the data workflow and graphical presentation; and implemented the analysis, the interpretation of data and wrote the manuscript. C.G. collected the data for cleaning, monitoring-sampling and monitoring-detection and revised the monitoring-sampling solutions; contributed to data analysis and conducting the manuscript; and discussed the results and revised and commented on the manuscript. K.K. collected the data for cleaning; revised these solutions according to the criteria; contributed to the analysis; homogenized the assignment of the TRLs; and discussed the results and revised and commented on the manuscript. J.M. collected the data for monitoring-detection; and discussed the results and revised and commented on the manuscript. J.C.-C. collected the data for monitoring-detection; and discussed the results and revised and commented on the manuscript. S.K. collected and revised the data for cleaning; and discussed the results and revised and commented on the manuscript. C.A.A.-G. collected the data on COBSEA and NOAA; and contributed to the analysis and discussed the results and commented on the manuscript. C.L. collected the data for monitoring-modelling, developed and applied the automated data workflow for a graphical representation of the data, contributed to conducting the manuscript; and analysed and discussed the results, and revised and commented on the manuscript.

\section{Competing interests}

The authors declare no competing interests.

\section{Additional information}

Supplementary information The online version contains supplementary material available at https://doi.org/10.1038/s41893-021-00726-2.

Correspondence and requests for materials should be addressed to N.B.

Peer review information Nature Sustainability thanks Jailson Moura and the other, anonymous, reviewer(s) for their contribution to the peer review of this work.

Reprints and permissions information is available at www.nature.com/reprints.

Publisher's note Springer Nature remains neutral with regard to jurisdictional claims in published maps and institutional affiliations.

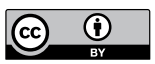

Open Access This article is licensed under a Creative Commons

Attribution 4.0 International License, which permits use, sharing, adaptation, distribution and reproduction in any medium or format, as long as you give appropriate credit to the original author(s) and the source, provide a link to the Creative Commons license, and indicate if changes were made. The images or other third party material in this article are included in the article's Creative Commons license, unless indicated otherwise in a credit line to the material. If material is not included in the article's Creative Commons license and your intended use is not permitted by statutory regulation or exceeds the permitted use, you will need to obtain permission directly from the copyright holder. To view a copy of this license, visit http://creativecommons. org/licenses/by/4.0/.

(c) The Author(s) 2021 